\title{
Re: Early Removal of Double-J Stents Decreases Urinary Tract Infections in Living Donor Renal Transplantation: A Prospective, Randomized Clinical Trial
}

\author{
Liu S1, Luo G1, Sun B1, Lu J1, Zu Q1', Yang S1, Zhang X1, Dong J2 \\ 'Chinese People's Liberation Army General Hospital, Department of Urology, Beijing, China \\ 2Hainan Branch of Chinese People's Liberation Army General Hospital, Department of Urology, Sanya, Hainan, China
}

Transplant Proc 2017;49:297-302. doi: 10.1016/j.transproceed.2016.12.007.

\section{EDITORIAL COMMENT}

Routine use and the duration of a double-J stent placement are controversial for renal transplant recipients. In this interim analysis of a double-blind, randomised controlled trial, differences in urological complications (e.g., urinary tract infections) between ureteral stent removal at 1 week and routine ureteral stent removal at 4 weeks in living kidney transplant recipients. In order to be able to perform power calculations on the incidence of major urologic complications, at least 271 patients are required in both groups. However, first 111 cases are described and minor urologic complications are given in this interim report. Stent removal was delayed in 3 patients due to increased serum creatinine levels and 5 patients were lost-to-follow-up due to loss of renal functions, thus 8 patients were excluded from the study. The patients were randomised to stent removal either at 1 week without local anesthesia by pulling the silk suture tied on the double-J catheter or cystoscopy or 4 weeks with cystoscopy under local anesthesia. All patients received intravenous antibiotics until 3 days posttransplant except co-trimoxazole. The 3-month incidence of urinary tract infection was 5.9\% and 29\% in the 1-week and 4-week stent removal groups, respectively. Also, there was a marked health care cost advantage of 1-week stent removal over 4-week stent removal because of the stent removal procedure and consumption of antibiotics.

Yarkın Kamil Yakupoğlu, MD

बCopyright 2017 by the Association of Urological Surgery / Journal of Urological Surgery published by Galenos Publishing House. 